\title{
Net-Clipping: An Approach to Deduce the Topology of Metal- Organic Frameworks Built with Zigzag Ligands
}

\author{
Borja Ortín-Rubio, ${ }^{\dagger}$ Hosein Ghasempour, ${ }^{\ddagger}$ Vincent Guillerm, ${ }^{* \dagger}$ Ali Morsali, ${ }^{\ddagger}$ Judith Juanhuix,,${ }^{\S}$ Inhar \\ Imaz, ${ }^{* \dagger}$ and Daniel Maspoch ${ }^{*+\perp}$ \\ ${ }^{\dagger}$ Catalan Institute of Nanoscience and Nanotechnology (ICN2), CSIC and Barcelona Institute of Science and \\ Technology, Campus UAB, Bellaterra, 08193 Barcelona, Spain. \\ ‡Department of Chemistry, Faculty of Sciences, Tarbiat Modares University, P. O. Box 14115-175, Tehran, Iran. \\ $\S$ Alba Synchrotron Light Facility, 08290 Cerdanyola del Vallès, Barcelona, Spain. \\ ${ }^{\perp}$ ICREA, Pg. Lluís Companys 23, Barcelona, 08010, Spain. \\ Supporting information for this article is given via a link at the end of the document \\ KEYWORDS. metal-organic framework $\bullet$ reticular chemistry $\bullet$ topology $\bullet$ zigzag ligand
}

\begin{abstract}
Herein we propose a new approach for deducing the topology of metal-organic frameworks (MOFs) assembled from organic ligands of low symmetry, which we call net-clipping. It is based on the construction of nets by rational deconstruction of edge-transitive nets comprising higher-connected molecular building blocks (MBBs). We have applied netclipping to predict the topologies of MOFs containing zigzag ligands. To this end, we derived 2-connected (2-c) zigzag ligands from 4-c square-like MBBs by first splitting the 4-c nodes into two 3-c nodes and then, clipping their two diagonally connecting groups. We demonstrate that, when this approach is applied to the 17 edge-transitive nets containing square-like 4-c MBBs, net-clipping deduces generation of ten nets with different underlying topologies. Moreover, we report that literature and experimental research corroborate successful implementation of our approach. As proof-of-concept, we employed netclipping to form three new MOFs built with zigzag ligands, each of which exhibits the deduced topology.
\end{abstract}

Reticular chemistry, defined as the "process of assembling judiciously designed rigid molecular building blocks (MBBs) into predetermined ordered structures (networks), which are held together by strong bonding", 1,2 has become essential in the design and synthesis of porous metal-organic frameworks (MOFs). Its success lies in precise analysis of the geometry and connectivity of the MBBs as well as in classification of their assemblies into different topologies. ${ }^{3}$ Thus, over the past two decades, application of the mathematic discipline of topology to $\mathrm{MBBs}^{4,5}$ [or secondary building units (SBUs)] ${ }^{2,6}$ has enabled synthesis of myriad MOFs based on reticulation of edgetransitive nets or their derived nets. Complementarily to these approaches, researchers have recently devised new design strategies to further expand rational design of MOFs, including supermolecular building blocks $(\mathrm{SBBs})^{7-9}$ and supermolecular building layers (SBLs). ${ }^{9,10}$ These strategies also include the merged-net approach, which is based on merging two edge-transitive nets into one minimal edgetransitive net, a useful strategy for rational design of mixedlinker MOFs. ${ }^{11}$

Herein we report a new design approach that, unlike the rational, bottom-up construction of edge-transitive nets, is based on the top-down deconstruction of edge-transitive nets. Our group recently reported that the combination of certain building blocks can induce structural irregularity (known as geometry mismatch) ${ }^{12}$ that complicates rational design of MOFs, as has been observed with use of lesssymmetric, 2-connected (2-c) groups such as bent, ${ }^{13}$ twisted 14 or zigzag ligands/MBBs. ${ }^{15}$ In addition, the various possibilities of orientation of non-linear ligands around inorganic MBBs lead to a high number of theoretical possibilities for polymorphism and therefore, a low structural predictability (Figure S1). ${ }^{16,17}$ However, as high symmetry would likely be mostly favored, we reasoned that less-symmetric ligands could be derived from moresymmetric MBBs of higher connectivity by simply reducing the connectivity of the latter. For example, a zigzag ligand can be formed by removing the two diagonally connecting groups of the two 3-c nodes derived from a 4-c MBB (Figure 1a). Accordingly, we reasoned that MOF structures made of less-symmetric ligands could be anticipated via rational clipping of the connecting groups of more-symmetric MBBs in edge-transitive nets. This new approach, which we have called net-clipping, provides further insights to our recent works on transversal reticular chemistry ${ }^{15}$ and geometry mismatch $^{12}$ and can facilitate rational design of MOFs built up from less-symmetric MBBs.

We propose use of net-clipping to rationalize/anticipate the MOFs that could be built from zigzag ligands. To this end, among the 54 edge-transitive nets (with D-symbol size $\leq 32$ ) reported by $0^{\prime}$ Keeffe et al., ${ }^{18}$ we first selected the 


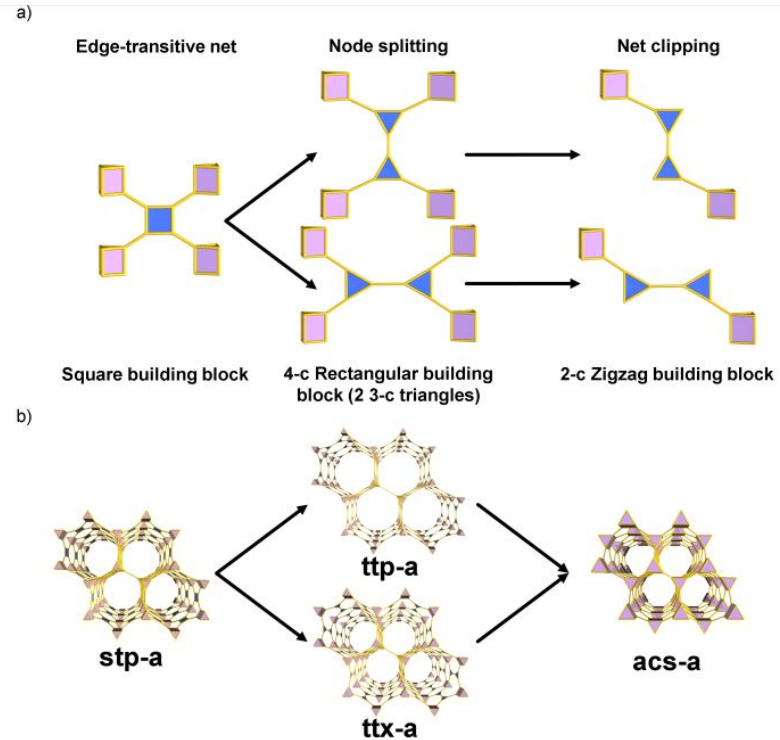

Figure 1. a) Schematic of the deconstruction of a 4-c square MBB in a zigzag building block by splitting the node into two 3-c triangles in different axes (node splitting), and then removing two diagonally connections (net-clipping). b) Schematic showing an example of our approach (node splitting + net clipping) applied to an edge-transitive net built from 4-c and 6-c triangular prism MBBs.

seventeen nets assembled from 4-c square-like MBBs. These nets are formed by combining a 4-c MBB with other polygonal and polyhedral MBBs (Table 1). Next, we derived these nets by splitting the 4-c nodes into two 3-c nodes (Figure 1). ${ }^{19}$ This node splitting step is important to reduce the symmetry of the 4-c MBBs and convert them into rectangular shapes, from which the two zigzag ligands can be originated by clipping the two diagonally connecting groups (Figure 1) ${ }^{20}$ Notably, this process led to 39 derived nets. ${ }^{21}$ Importantly, reducing the symmetry of some of the initial edge-transitive nets (nbo, ssb, pts, scu and ftw) leads to two-symmetrically different 4-c planar nodes. In these cases, as the two types of nodes can be split distinctly, more than two derived nets can be formed.

We then applied net-clipping to the derived nets by erasing the two diagonally connecting groups to mimic the presence of a zigzag-shaped MBBs (Figure 1b; Figures S4S20). The ten resultant nets are summarized in Table 1 . We concluded that most 3D nets (pto, ssb, pts, pth, she, soc, stp, scu and ftw) are clipped into other 3D nets (srs, lvt, dia, qtz, hxg, crs, acs, bcu and fcu, respectively); that some 3D nets (nbo, lvt and ssb) are clipped into the 2D sql net; and that the remaining nets (tbo, rhr, ssa, sqc, csq and shp) cannot be clipped into other nets. Interestingly, we found a common feature among all these latter edge-transitive nets: the presence of a 6-cycle ${ }^{22}$ that comprises three 3-c nodes (derived from three 4-c nodes) and three other MBBs and that frustrates the net clipping in a fully zigzag fashion (Figures S21,S22).

Once we had theoretically deduced the MOF structures that could be formed using zigzag ligands, we experimentally assessed our net-clipping approach. To this
Table 1. Net-clipping of all the derived nets from 4-c square nodes in the 17 selected edge-transitive nets.

\begin{tabular}{|c|c|c|c|}
\hline MBB & Main Topology & Derived Net & $\begin{array}{c}\text { Clipped } \\
\text { Net }\end{array}$ \\
\hline & tbo & $\begin{array}{l}\text { tbd } \\
\text { xaa }\end{array}$ & \\
\hline & pto & ptd & srs \\
\hline & nbo & $\begin{array}{l}\text { fof } \\
\text { fog } \\
\text { tfb }\end{array}$ & sql \\
\hline & rhr & $\begin{array}{c}\text { ucp } \\
\text { sqc12288* }\end{array}$ & \\
\hline & lvt & $\begin{array}{c}\text { lil } \\
\text { lim }\end{array}$ & sql \\
\hline & ssa & $\begin{array}{c}\text { sty } \\
\text { Initial } \\
\text { structure; } \\
\text { Bond sets: } \\
\text { 1,3:bbp** }\end{array}$ & \\
\hline & $s s b$ & $\begin{array}{l}\text { stu } \\
\text { stw }\end{array}$ & sql \\
\hline & & $\begin{array}{l}\text { stj } \\
\text { stx }\end{array}$ & lvt \\
\hline & pts & $\begin{array}{c}\text { dmd } \\
\text { dmg } \\
\text { dmh } \\
\text { tfi }\end{array}$ & dia \\
\hline & pth & $\begin{array}{c}\text { hst } \\
3,4 \mathrm{~T} 45^{* * *} \\
\end{array}$ & qtz \\
\hline & she & sqc12215* & hxg \\
\hline & soc & $\begin{array}{l}\text { cdj } \\
\text { edq }\end{array}$ & crs \\
\hline & stp & $\begin{array}{l}\text { ttp } \\
\mathrm{ttx}\end{array}$ & acs \\
\hline & scu & $\begin{array}{c}\text { tty } \\
\text { cut } \\
3,3,8 \mathrm{~T} 132^{* * *} \\
\end{array}$ & bcu \\
\hline & sqc & $\begin{array}{l}\operatorname{sqc} 3520^{*} \\
\operatorname{sqc} 3782^{*}\end{array}$ & \\
\hline & $\operatorname{csq}$ & $\begin{array}{l}\text { xly } \\
\text { xlz }\end{array}$ & \\
\hline & ftw & $\begin{array}{l}\text { kle } \\
\text { kxe } \\
\text { ttv }\end{array}$ & fcu \\
\hline & shp & $\begin{array}{l}\text { ced } \\
\text { cec }\end{array}$ & \\
\hline
\end{tabular}

* Topologies corresponding to the Systre code in the Epinet database.

** Topology corresponding to the subnet transformation symbols nomenclature.

*** Topologies corresponding to the TOPOS symbols nomenclature.

end, we chose two types of MOFs assembled from combining a 4-c MBB with a 4-c square-like MBB or a 12-c cuboctahedral MBB. Then, we combined the zigzag ligand analogs (derived from the 4-c MBB) with the corresponding 
polyhedral MBBs to synthesize two new MOFs, whose topologies we compared with those that we had deduced by net-clipping. Note that, in two other cases, to further support the net-clipping approach, we used MOF structures already reported in the literature (MOFs made by combining a 4-c MBB with 4-c tetrahedral or 8-c cubic MBBs).

We began with the nbo MOF PCN-10 (derived net: fof), which is built by connecting 4-c square-like $\mathrm{Cu}(\mathrm{II})$ paddlewheel MBBs through 4-c 3,3',5,5'-azobenzene- tetracarboxylate $\left(3,3^{\prime}, 5,5^{\prime}\right.$-ABTC) ligands. ${ }^{23}$ In this case, netclipping deduced the formation of a 2D sql MOF (Table 1). Remarkably, replacing 3,3',5,5'-ABTC with the corresponding zigzag 3,3'-azobenzene-dicarboxylate (3,3'ABDC) ligand, afforded the expected 2D sql MOF (Figure $2 \mathrm{a}$,top). This entailed reaction of copper(II) nitrate salt and $\mathrm{H}_{2}\left(3,3^{\prime}\right.$-ABDC) in $\mathrm{N}, \mathrm{N}$-dimethylformamide (DMF) under solvothermal conditions, which yielded green needleshaped crystals of $\mathrm{Cu}$-sql-3,3'-ABDC. Single-crystal X-ray diffraction (SCXRD) revealed formation of a ABCD packing

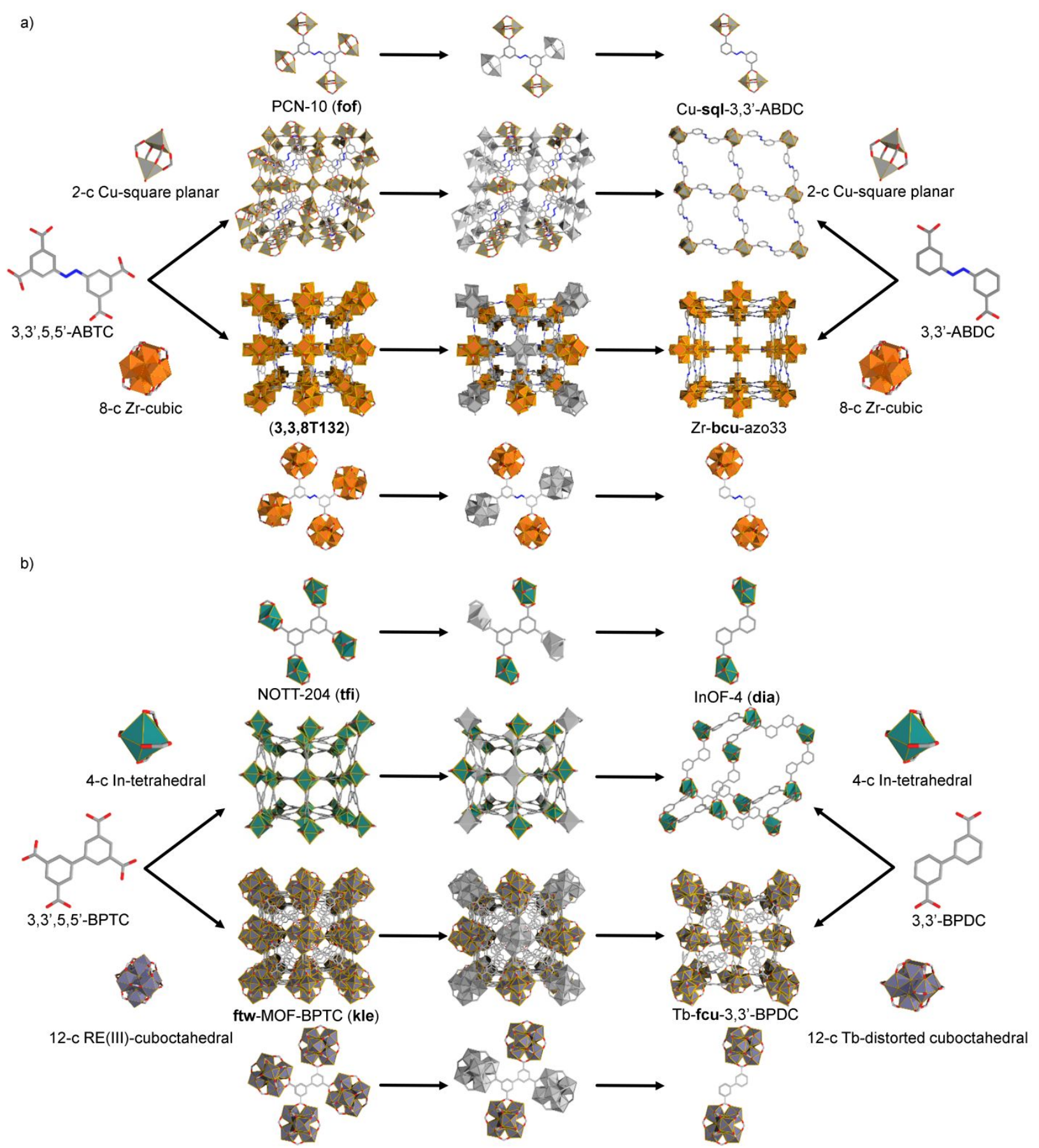

Figure 2. Schematic of the net-clipping approach applied to formation of MOFs from (a) 3,3',5,5'-ABTC ligand to zigzag 3,3'ABDC combined with (top) 4-c paddle-wheel Cu(II) MBBs and (bottom) 8-c cubic Zr(IV)-based MBBs; and from (b) 3,3',5,5'BPTC to 3,3'-BPDC ligand combined with (top) 4-c tetrahedral In(III)-based MBBs and (bottom) 12-c cuboctahedral Tb(III)based MBBs. 
of a 2D-network of formula $\mathrm{Cu}_{2}\left(3,3^{\prime}-\mathrm{ABDC}\right)_{2}\left(\mathrm{H}_{2} \mathrm{O}\right)_{2}$, which crystallizes in the $C 2 / m$ space group (Figure 2 ). As expected, the building unit in $\mathrm{Cu}-\mathbf{s q l}-3,3^{\prime}-\mathrm{ABDC}$ is the $\mathrm{Cu}$ (II) paddlewheel unit. In this framework, each of these units is connected to four others through four bridging zigzag 3,3'ABDC ligands, adopting a 4-c sql underlying topology (Figure 2a,top).

Interestingly, our net-clipping approach is further corroborated by the fact that an isostructural sql MOF made by linking Zn(II) paddle-wheel units by 3,3'-ABDC ligands had previously been described by Liang et al. ${ }^{24}$ Similarly, two other independently, previously reported structures reinforce our approach: NOTT-204,25 a pts MOF (derived net: tfi) built by linking the 4-c 3,3',5,5'-biphenyltetracarboxylate $\left(3,3^{\prime}, 5,5^{\prime}-\mathrm{BPTC}\right)$ ligand and the 4-c tetrahedral In(III)-based MBB; and InOF- $4,{ }^{26}$ a dia-MOF made of connecting the same 4-c tetrahedral In(III)-based MBBs through the zigzag 3,3'-biphenyl-dicarboxylate $(3,3$ 'BPDC) ligand. Interestingly, both of these MOF structures are related by net-clipping, which deduced formation of a clipped dia topology from a pts topology (Table 1 and Figure 2b,top).

Next, we shifted our attention to another MOF assembled from the 4-c 3,3',5,5'-ABTC ligand and a higher-connected $\mathrm{MBB}$, the 8-c cubic $\mathrm{Zr}_{6} \mathrm{O}_{4}(\mathrm{OH})_{4}(\mathrm{OOC}-\mathrm{R})_{8}\left(\mathrm{H}_{2} \mathrm{O}\right)_{4}(\mathrm{OH})_{4}$ hexanuclear MBB. ${ }^{27}$ This MOF shows the scu topology, in which we reasoned that replacement of 3,3',5,5'-ABTC with 3,3'-ABDC would generate a clipped bcu MOF (Table 1). Interestingly, our group recently reported that combination of this 8-c MBB with a series of zigzag ligands, including 3,3'ABDC, leads to formation of MOFs with the bcu topology (Figure 2a,bottom), which further supports net-clipping. ${ }^{15}$

Recently, Eddaoudi et al. reported that combining 4-c ligands (e.g. 3,3',5,5'-ABTC or 3,3',5,5'-BPTC) with 12-c cuboctahedral rare earth metal (RE) MBBs affords RE-ftwMOFs (derived net: kle). ${ }^{28}$ From this topology, net-clipping

a)

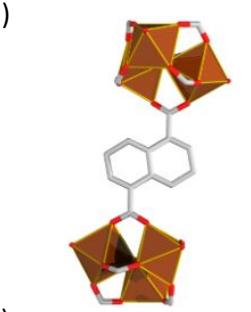

b)

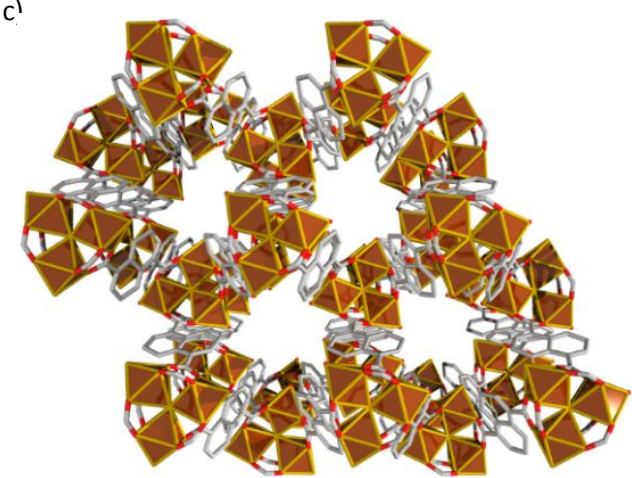

Figure 3. Crystal structure of Fe-acs-1,5-NDC, showing a) the zigzag connection between the Fe-trimers; b) the trigonal bipyramid cage; and c) the channels formed through the $c$ axis. predicts formation of a MOF with the fcu underlying topology. To investigate this, we used the zigzag 3,3'-BPDC ligand as a substitute for the 4-c 3,3',5,5'-BPTC ligand. Reaction of terbium(III) nitrate salt and $\mathrm{H}_{2}\left(3,3^{\prime}\right.$-BPDC) in the presence of 2-fluorobenzoic acid in DMF under solvothermal conditions yielded transparent octahedral crystals of Tb-fcu-3,3'-BPDC. SCXRD revealed formation of a 3D net with formula $\left[\left(\mathrm{CH}_{3}\right)_{2} \mathrm{NH}_{2}\right]_{2}\left[\mathrm{~Tb}_{6}\left(\mu_{3}-\mathrm{OH}\right)_{8}\left(3,3^{\prime}-\right.\right.$ BPDC $\left.)_{6}\left(\mathrm{H}_{2} \mathrm{O}\right)_{4}\right]$, which crystallizes in the $P 2_{1} / n$ space group. As we had expected, the presence of 2-fluorobenzoic acid as modulator $^{29}$ enabled formation of the hexanuclear RE MBB in Tb-fcu-3,3'-BPDC. In this framework, each of these MBBs is connected to twelve others, through twelve bridging zigzag 3,3'-BPDC groups, adopting overall a 12-c fcu topology (Figure 2b, bottom). Note that, compared to the archetypical 12-c Zr-fcu-4,4'-BPDC (known as UiO-67), ${ }^{30}$ Tb-fcu-3,3'-BPDC shows a less-symmetric, distorted structure. We attributed this feature to the transversal parameter of the zigzag ligand as well as to the different metal-based MBBs, in which Zr(IV) ions had been replaced with $\mathrm{Tb}$ (III) ions, thereby a slightly different coordination environment (Figures S30,S31).

Once we had confirmed the feasibility of our net-clipping approach, we applied it to synthesize a new acs-MOF built with a zigzag ligand. To this end, we synthesized a rigid zigzag 1,5-naphtalenedicarboxate (1,5-NDC) ligand and selected the well-known $[\mathrm{Fe}(\mathrm{III})]_{3} \mathrm{O}$ trimeric unit as the trigonal prism MBB. ${ }^{31}$ We synthesized this zigzag ligand because, to our knowledge, a stp MOF assembled from the corresponding 4-c 1,4,5,8-naphtalenetetracarboxylate ligand and a 6-c trigonal prism MBB - for which net-clipping deduced formation of an acs topology - have never previously been reported. First, $\mathrm{H}_{2}(1,5-\mathrm{NDC})$ was synthesized from the corresponding diamine-derivative via several functional group interconversions (see SI). Then, the Fe(III) trimeric unit was synthesized in an acetic acid solution, according to a literature protocol. ${ }^{32}$ Finally, the pre-formed $\mathrm{Fe}(\mathrm{III})$ unit was reacted with $\mathrm{H}_{2}(1,5-\mathrm{NDC})$ and acetic acid in DMF under solvothermal conditions for $48 \mathrm{~h}$. After this period, orange hexagonal crystals suitable for SCXRD were collected. SCXRD revealed formation of a 3D structure with formula $\mathrm{Fe}_{3}\left(\mu_{3}-\mathrm{O}\right)(1,5-\mathrm{NDC})_{3}\left(\mathrm{H}_{2} \mathrm{O}\right)_{2}(\mathrm{OH})$, which crystallizes in the $P-6_{3} m$ space group. In Fe-acs-1,5NDC, each trimer is connected to six others through six zigzag 1,5-NDC ligands, adopting the 6-c acs underlying topology (Figure 3). Note that the structure of Fe-acs-1,5NDC, although slightly distorted, is similar to that of MOF235/MIL-88B, ${ }^{33,34}$ which also exhibits an underlying acs topology.

In summary, we have proposed and validated a new approach, net-clipping, for rational design of MOFs made of zigzag ligands. First, we demonstrated the relationship between these ligands and more symmetric 4-c ligands. Next, we studied the edge-transitive nets with 4-c nodes with associated square vertex figure, and their derived nets, to identify the possible outcomes. Then, we applied our netclipping approach to deduce the different topologies that should be accessible upon assembly of zigzag ligands with different polyhedral MBBs. Finally, we demonstrated the feasibility of net-clipping through the successful design and assembly of three novel MOFs based on MBBs with different 
connectivities: $\mathrm{Cu}$-sql-3,3'-ABDC (4-c, paddle wheel), Feacs-1,5-NDC (6-c, trimer) and Tb-fcu-3,3'-BPDC (12-c, hexamer). Our approach enriches the repertoire for topological predictions, and we anticipate the application of net-clipping to bent ligands, via clipping of 4-c MBBs in other ways, as well as its eventual use with MBBs of other connectivity.

\section{ASSOCIATED CONTENT}

The Supporting Information is available free of charge via the Internet at http://pubs.acs.org.

Chemicals, instrumentation, net-clipping approach and synthetic procedures, schemes of the topologies resulting from the net-clipping, PXRD, crystallographic data, structural details and ${ }^{1} \mathrm{H}$ NMR.

\section{AUTHOR INFORMATION}

\section{Corresponding Author}

Vincent Guillerm - Catalan Institute of Nanoscience and Nanotechnology (ICN2), CSIC and Barcelona Institute of Science and Technology, Campus UAB, Bellaterra, 08193 Barcelona, Spain; E-mail: vguillerm@protonmail.com Present address: Functional Materials Design, Discovery \& Development Research Group (FMD $\left.{ }^{3}\right)$, Advanced Membranes \& Porous Materials Center, Division of Physical Sciences and Engineering, King Abdullah University of Science and Technology (KAUST), Thuwal 23955-6900, Kingdom of Saudi Arabia.

Inhar Imaz - Catalan Institute of Nanoscience and Nanotechnology (ICN2), CSIC and Barcelona Institute of Science and Technology, Campus UAB, Bellaterra, 08193 Barcelona, Spain; E-mail: inhar.imaz@icn2.cat

Daniel Maspoch - Catalan Institute of Nanoscience and Nanotechnology (ICN2), CSIC and Barcelona Institute of Science and Technology, Campus UAB, Bellaterra, 08193 Barcelona, Spain; ICREA, Pg. Lluís Companys 23, Barcelona, 08010, Spain; E-mail: daniel.maspoch@icn2.cat

\section{Authors}

Borja Ortín-Rubio - Catalan Institute of Nanoscience and Nanotechnology (ICN2), CSIC and Barcelona Institute of Science and Technology, Campus UAB, Bellaterra, 08193 Barcelona, Spain.

Hosein Ghasempour - Department of Chemistry, Faculty of Sciences, Tarbiat Modares University, P. O. Box 14115-175, Tehran, Iran.

Ali Morsali - Department of Chemistry, Faculty of Sciences, Tarbiat Modares University, P. O. Box 14115-175, Tehran, Iran.

Judith Juanhuix - Alba Synchrotron Light Facility, 08290 Cerdanyola del Vallès, Barcelona, Spain.

\section{ACKNOWLEDGMENT}

This work was supported by the Spanish MINECO (project RTI2018-095622-B-I00), the Catalan AGAUR (project 2017
SGR 238), and the ERC under the EU-FP7 (ERC-Co 615954). It was also funded by the CERCA Program/Generalitat de Catalunya. ICN2 is supported by the Severo Ochoa program from the Spanish MINECO (Grant No. SEV-2017-0706).

\section{REFERENCES}

(1) Yaghi, O. M.; Kalmutzki, M. J.; Diercks, C. S. Introduction to Reticular Chemistry; Wiley, 2019.

(2) Yaghi, O. M.; O’Keeffe, M.; Ockwig, N. W.; Chae, H. K.; Eddaoudi, M.; Kim, J. Reticular Synthesis and the Design of New Materials. Nature, 2003, 423, 705-714.

(3) O’Keeffe, M.; Peskov, M. A.; Ramsden, S. J.; Yaghi, O. M. The Reticular Chemistry Structure Resource (RCSR) Database of, and Symbols for, Crystal Nets. Acc. Chem. Res. 2008, 41, 1782-1789.

(4) Stein, A.; Keller, S. W.; Mallouk, T. E. Turning down the Heat: Design and Mechanism in Solid-State Synthesis. Science 1993, 259, $1558-1564$

(5) Eddaoudi, M.; Moler, D. B.; Li, H.; Chen, B.; Reineke, T. M.; O'Keeffe, M.; Yaghi, O. M. Modular Chemistry: Secondary Building Units as a Basis for the Design of Highly Porous and Robust MetalOrganic Carboxylate Frameworks. Acc. Chem. Res. 2001, 34, 319 330.

(6) O'Keeffe, M.; Yaghi, O. M. Deconstructing the Crystal Structures of Metal-Organic Frameworks and Related Materials into Their Underlying Nets. Chem. Rev. 2012, 112, 675-702.

(7) Nouar, F.; Eubank, J. F.; Bousquet, T.; Wojtas, L.; Zaworotko, M. J.; Eddaoudi, M. Supermolecular Building Blocks (SBBs) for the Design and Synthesis of Highly Porous Metal-Organic Frameworks. J. Am. Chem. Soc. 2008, 130, 1833-1835.

(8) Perry VI, J. J.; Perman, J. A.; Zaworotko, M. J. Design and Synthesis of Metal-Organic Frameworks Using Metal-Organic Polyhedra as Supermolecular Building Blocks. Chem. Soc. Rev. 2009, 38, 1400-1417.

(9) Guillerm, V.; Kim, D.; Eubank, J. F.; Luebke, R.; Liu, X.; Adil, K.; Lah, M. S.; Eddaoudi, M. A Supermolecular Building Approach for the Design and Construction of Metal-Organic Frameworks. Chem. Soc. Rev. 2014, 43, 6141-6172.

(10) Eubank, J. F.; Mouttaki, H.; Cairns, A. J.; Belmabkhout, Y.; Wojtas, L.; Luebke, R.; Alkordi, M.; Eddaoudi, M. The Quest for Modular Nanocages: tbo-MOF as an Archetype for Mutual Substitution, Functionalization, and Expansion of Quadrangular Pillar Building Blocks. J. Am. Chem. Soc. 2011, 133, 14204-14207.

(11) Jiang, H.; Jia, J.; Shkurenko, A.; Chen, Z.; Adil, K.; Belmabkhout, Y.; Weselinski, L. J.; Assen, A. H.; Xue, D. X.; O’Keeffe, M.; Eddaoudi, M. Enriching the Reticular Chemistry Repertoire: Merged Nets Approach for the Rational Design of Intricate MixedLinker Metal-Organic Framework Platforms. J. Am. Chem. Soc. 2018, 140, 8858-8867.

(12) Guillerm, V.; Maspoch, D. Geometry Mismatch and Reticular Chemistry: Strategies to Assemble Metal-Organic Frameworks with Non-Default Topologies. J. Am. Chem. Soc. 2019, 141, 1651716538.

(13) Eddaoudi, M.; Kim, J.; Vodak, D.; Sudik, A.; Wachter, J.; O'Keeffe, M.; Yaghi, O. M. Geometric Requirements and Examples of Important Structures in the Assembly of Square Building Blocks. Proc. Natl. Acad. Sci. U. S. A. 2002, 99, 4900-4904.

(14) Eddaoudi, M.; Kim, J.; O’Keeffe, M.; Yaghi, O. M. $\mathrm{Cu}_{2}[o-\mathrm{Br}$ $\left.\mathrm{C}_{6} \mathrm{H}_{3}\left(\mathrm{CO}_{2}\right)_{2}\right]_{2}\left(\mathrm{H}_{2} \mathrm{O}\right)_{2} \cdot(\mathrm{DMF})_{8}\left(\mathrm{H}_{2} \mathrm{O}\right)_{2}$ : A Framework Deliberately Designed to Have the NbO Structure Type. J. Am. Chem. Soc. 2002, 124, 376-377.

(15) Guillerm, V.; Grancha, T.; Imaz, I.; Juanhuix, J.; Maspoch, D. Zigzag Ligands for Transversal Design in Reticular Chemistry: Unveiling New Structural Opportunities for Metal-Organic Frameworks. J. Am. Chem. Soc. 2018, 140, 10153-10157.

(16) Bon, V.; Senkovska, I.; Baburin, I. A.; Kaskel, S. Zr- and HfBased Metal-Organic Frameworks: Tracking down the Polymorphism. Cryst. Growth Des. 2013, 13, 1231-1237. 
(17) Drache, F.; Bon, V.; Senkovska, I.; Getzschmann, J.; Kaskel, S. The Modulator Driven Polymorphism of $\mathrm{Zr}(\mathrm{IV})$ Based MetalOrganic Frameworks. Philos. Trans. R. Soc. A Math. Phys. Eng. Sci. 2017, 375, 20160027.

(18) Delgado-Friedrichs, O.; O’Keeffe, M. Three-Periodic Tilings and Nets: Face-Transitive Tilings and Edge-Transitive Nets Revisited. Acta Crystallogr. Sect. A Found. Crystallogr. 2007, 63, 344-347.

(19) Eubank, J. F.; Walsh, R. D.; Poddar, P.; Srikanth, H.; Larsen, R. W.; Eddaoudi, M. Metal-Organic Framework Diversity via Heterocoordination of a Multifunctional Ligand: $\mathrm{SrAl}_{2}$ and a Novel (3,4)-Connected Network. Cryst. Growth Des. 2006, 6, 1453-1457.

(20) Luebke, R.; Belmabkhout, Y.; Weseliński, Ł. J.; Cairns, A. J.; Alkordi, M.; Norton, G.; Wojtas, Ł.; Adil, K.; Eddaoudi, M. Versatile Rare Earth Hexanuclear Clusters for the Design and Synthesis of Highly-Connected ftw -MOFs. Chem. Sci. 2015, 6, 4095-4102.

(21) Li, M.; Li, D.; O’Keeffe, M.; Yaghi, O. M. Topological Analysis of Metal-Organic Frameworks with Polytopic Linkers and/or Multiple Building Units and the Minimal Transitivity Principle. Chem. Rev. 2014, 114, 1343-1370.

(22) Blatov, V. A.; O'Keeffe, M.; Proserpio, D. M. Vertex-, Face-, Point-, Schläfli-, and Delaney-Symbols in Nets, Polyhedra and Tilings: Recommended Terminology. CrystEng Comm 2010, 12, 4448.

(23) Wang, X. Sen; Shengqian, M.; Rauch, K.; Simmons, J. M.; Yuan, D.; Wang, X.; Yildirim, T.; Cole, W. C.; López, J. J.; De Meijere, A.; Zhou, H. C. Metal-Organic Frameworks Based on Double-BondCoupled Di-Isophthalate Linkers with High Hydrogen and Methane Uptakes. Chem. Mater. 2008, 20, 3145-3152.

(24) Chen, Z. F.; Zhang, Z. L.; Tan, Y. H.; Tang, Y. Z.; Fun, H. K.; Zhou, Z. Y.; Abrahams, B. F.; Liang, H. Coordination Polymers Constructed by Linking Metal Ions with Azodibenzoate Anions. CrystEngComm 2008, 10, 217-231.

(25) Yang, S.; Callear, S. K.; Ramirez-Cuesta, A. J.; David, W. I. F.; Sun, J.; Blake, A. J.; Champness, N. R.; Schröder, M. Pore with Gate: Modulating Hydrogen Storage in Metal-Organic Framework Materials via Cation Exchange. Faraday Discuss. 2011, 151, 19-36.

(26) Qian, J.; Jiang, F.; Su, K.; Li, Q.; Zhou, K.; Wu, M.; Yuan, D.; Hong, M. Sorption Comparison of Two Indium-Organic Framework

TOC

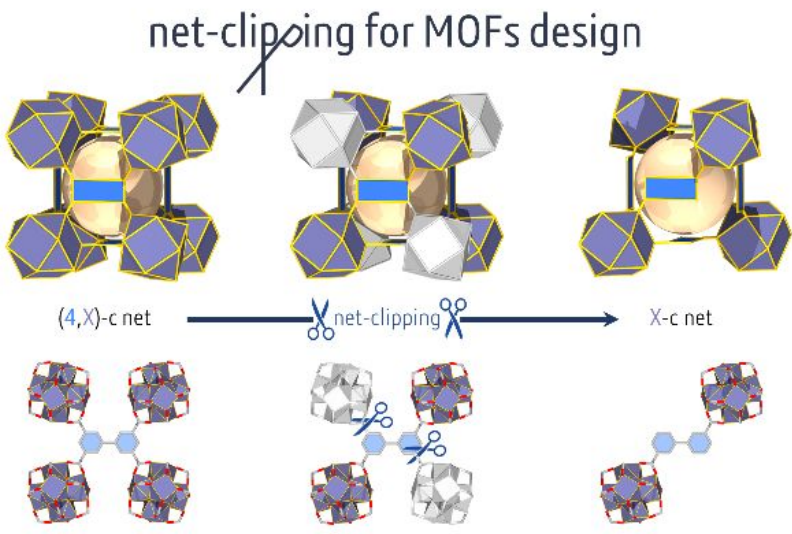

Isomers with Syn-Anti Configurations. CrystEngComm 2014, 16, 7434-7439.

(27) Wang, H.; Dong, X.; Lin, J.; Teat, S. J.; Jensen, S.; Cure, J.; Alexandrov, E. V.; Xia, Q.; Tan, K.; Wang, Q.; Olson, D. H.; Proserpio, D. M.; Chabal, Y. J.; Thonhauser, T.; Sun, J.; Han, Y.; Li, J. Topologically Guided Tuning of Zr-MOF Pore Structures for Highly Selective Separation of C6 Alkane Isomers. Nat. Commun. 2018, 9, $1-11$.

(28) Xue, D. X.; Cadiau, A.; Weseliński, Ł. J.; Jiang, H.; Bhatt, P. M.; Shkurenko, A.; Wojtas, L.; Zhijie, C.; Belmabkhout, Y.; Adil, K.; Eddaoudi, M. Topology Meets MOF Chemistry for Pore-Aperture Fine Tuning: ftw-MOF Platform for Energy-Efficient Separations: Via Adsorption Kinetics or Molecular Sieving. Chem. Commun. 2018, 54, 6404-6407.

(29) Xue, D. X.; Cairns, A. J.; Belmabkhout, Y.; Wojtas, L.; Liu, Y.; Alkordi, M. H.; Eddaoudi, M. Tunable Rare-Earth fcu-MOFs: A Platform for Systematic Enhancement of $\mathrm{CO}_{2}$ Adsorption Energetics and Uptake. J. Am. Chem. Soc. 2013, 135, 7660-7667.

(30) Cavka, J. H.; Jakobsen, S.; Olsbye, U.; Guillou, N.; Lamberti, C.; Bordiga, S.; Lillerud, K. P. A New Zirconium Inorganic Building Brick Forming Metal Organic Frameworks with Exceptional Stability. J. Am. Chem. Soc. 2008, 130, 13850-13851.

(31) Schoedel, A.; Zaworotko, M. J. $\left[\mathrm{M}_{3}\left(\mathrm{M}_{3}-\mathrm{O}\right)\left(\mathrm{O}_{2} \mathrm{CR}\right)_{6}\right]$ and Related Trigonal Prisms: Versatile Molecular Building Blocks for Crystal Engineering of Metal-Organic Material Platforms. Chem. Sci. 2014, 5, 1269-1282.

(32) Feng, D.; Wang, K.; Wei, Z.; Chen, Y. P.; Simon, C. M.; Arvapally, R. K.; Martin, R. L.; Bosch, M.; Liu, T. F.; Fordham, S.; Yuan, D.; Omary, M. A.; Haranczyk, M.; Smit, B.; Zhou, H. C. Kinetically Tuned Dimensional Augmentation as a Versatile Synthetic Route towards Robust Metal-Organic Frameworks. Nat. Commun. 2014, $5,1-9$.

(33) Sudik, A. C.; Côté, A. P.; Yaghi, O. M. Metal-Organic Frameworks Based on Trigonal Prismatic Building Blocks and the New "acs" Topology. Inorg. Chem. 2005, 44, 2998-3000.

(34) Surblé, S.; Serre, C.; Mellot-Draznieks, C.; Millange, F.; Férey, G. A New Isoreticular Class of Metal-Organic-Frameworks with the MIL-88 Topology. Chem. Commun. 2006, 284-286. 